\title{
NOUVELle
}

\section{La fonction d'un long ARN non codant décodée dans la fibrose pulmonaire idiopathique}

Grégoire Savary ${ }^{1,2}$, Nicolas Pottier ${ }^{2,3}$, Bernard Mari ${ }^{1}$, Christelle Cauffiez $^{2}$
'Université Côte d'Azur, CNRS, IPMC, FHU-OncoAge, Valbonne, France.

${ }^{2}$ Université de Lille, EA 4483-IMPECS, Lille, France.

${ }^{3}$ Centre hospitalo-universitaire de Lille, Service de toxicologie et génopathies, Lille, France.

mari@unice.fr
Les ARN non codants: de nouvelles cibles thérapeutiques

Les recherches sur les ARN non codants (ARNnc) et leurs rôles biologiques ont révolutionné notre conception du génome et nos connaissances sur la régulation de l'expression génique. II est maintenant établi que la dérégulation des ARNnc joue un rôle central dans le début et la progression de la plupart des maladies complexes [1]. Ces ARNnc comprennent, d'une part, des ARN régulateurs assez bien caractérisés comme les microARN (miARN) et les piwi-interacting RNA (piARN) et, d'autre part, des classes d'ARNnc moins bien connues telles que les longs ARN non codants (IncARN).

Les miARN, dont plus de 2500 ont été répertoriés chez l'homme, sont des petits ARN d'environ 20 nucléotides agissant en tant que régulateurs négatifs de l'expression génique capables soit d'inhiber la traduction soit d'induire la dégradation de leurs ARN messagers cibles [2] $(\rightarrow)$

$\rightarrow$ Voir la Synthèse de C. Hartman et al., $\mathrm{m} / \mathrm{s} \mathrm{n}{ }^{\circ} 10$, mars 2004, page 894

Les IncARN, quant à eux, sont des transcrits de plus de 200 nucléotides impliqués dans de très nombreux mécanismes de régulation transcriptionnelle et posttranscriptionnelle [3] $(\rightarrow)$

$(\rightarrow)$ Voir la Synthèse de T. Pedrazzini, $m / s$ $n^{\circ} 3$, mars 2015, page 261

Tous ces ARN non codants représentent, par leurs mécanismes d'action et du fait de leur perturbation dans la majorité des processus pathologiques, de nouvelles cibles thérapeutiques potentielles, et certains essais cliniques sont d'ores et déjà en cours $[4,5]$.
La fibrose pulmonaire idiopathique La fibrose pulmonaire idiopathique (FPI) est une maladie chronique irréversible affectant l'interstitium pulmonaire, dont la médiane de survie après diagnostic est inférieure à 5 ans, et pour laquelle les options thérapeutiques sont limitées [6]. La physiopathologie de la FPI est particulièrement complexe, et implique notamment une activation excessive des fibroblastes pulmonaires en myofibroblastes suite à l'altération des cellules épithéliales alvéolaires en réponse à une agression chronique, dont l'origine reste inconnue dans la majorité des cas. La fibrose se caractérise par une accumulation de composants de la matrice extracellulaire sécrétés par les myofibroblastes, à l'origine de la destruction de l'architecture tissulaire.

Sur le plan moléculaire, il est maintenant établi que le transforming growth factor $\beta$ (TGF- $\beta$ ) joue un rôle majeur dans l'apparition et la progression de la fibrose en favorisant le recrutement, la prolifération et l'activation des fibroblastes pulmonaires [7]. Bien que l'inhibition du TGF- $\beta$ représente une stratégie thérapeutique séduisante, cette approche s'est finalement révélée décevante, car l'administration d'inhibiteurs du TGF- $\beta$ entraîne de très nombreux effets indésirables dus à l'action de ce facteur de croissance sur les cellules d'autres organes ou tissus. De ce fait, l'identification de nouvelles options thérapeutiques visant à inhiber sélectivement la voie du TGF- $\beta$ dans les myofibroblastes fait l'objet de nombreuses recherches.
DNM30S, un IncARN profibrotique spécifiquement exprimé dans les myofibroblastes

Nous avions précédemment montré le rôle majeur joué par un «FibromiR », miR-199a-5p, dans le contrôle de l'activation des myofibroblastes dans la FPI $[8,9](\rightarrow)$

$(\rightarrow)$ Voir la Nouvelle de I.S. Hénaoui, $m / s n^{\circ} 5$, mai 2013 , MiR-199a-5p, en page 461 régulant l'expression du gène codant la cavéoline-1 (CAVI), est un élément essentiel de la réponse des fibroblastes pulmonaires au TGF- $\beta$ (Figure 1). En effet, CAV1, une protéine membranaire essentielle à la formation des cavéoles, est impliquée dans l'internalisation et la dégradation du complexe formé par le TGF- $\beta$ et ses récepteurs. Nous avons maintenant analysé le profil des IncARN dont la synthèse varie au cours de la différenciation des fibroblastes en myofibroblastes induite par le TGF- $\beta$. Cette analyse nous a permis d'identifier DNM30S (dynamin 3 opposite strand), un IncARN fortement induit dans ces conditions expérimentales. Nous avons montré son rôle clé dans la voie de signalisation du TGF- $\beta$, ainsi que son expression spécifique dans les myofibroblastes pulmonaires à l'aide d'un modèle murin de fibrose pulmonaire induite par la bléomycine. Sa surexpression exclusivement dans les myofibroblastes pulmonaires de patients atteints de FPI a ensuite été confirmée [10].

DNM30S est un transcrit polycistronique précurseur de trois miARN matures: miR-199a-5p, préalablement caractérisé par l'équipe [9], ainsi que miR- 


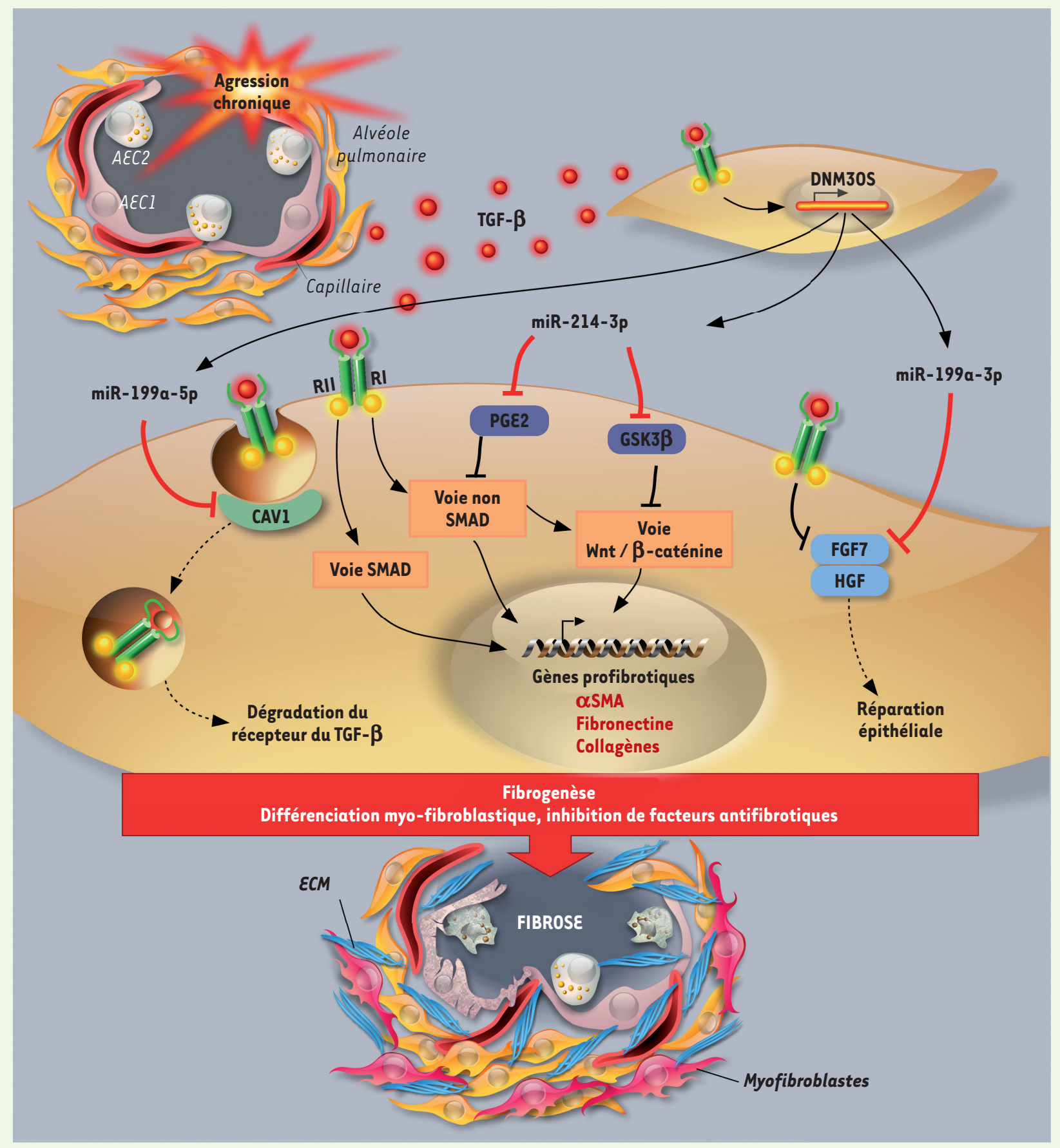

Figure 1. Modèle résumant les effets profibrotiques de DNM30S lors du processus de fibrose pulmonaire. L'agression chronique de l'épithélium alvéolaire induit un relargage du TGF- $\beta$ (transforming growth factor $\beta$ ) à l'origine de la surexpression de DNM30S (dynamin 3 opposite strand) et des trois miARN associés, miR-199a-5p, miR-214-3p et miR-199a-3p. En ciblant CAV1 (cavéoline-1), miR-199a-5p amplifie la signalisation du TGF- $\beta$, dépendante et indépendante des facteurs de transcription Smad, en limitant la voie de dégradation des récepteurs du TGF- $\beta$. MiR-214-3p est, quant à lui, impliqué dans l'activation de la voie du TGF- $\beta$ indépendante des Smad en inhibant la production de prostaglandine $\varepsilon 2$ (PGE2) via le ciblage de COX-2 (cyclooxygénase 2) et en activant la voie Wnt / $\beta$-caténine par l'inhibition d'une deuxième cible, GSK3 $\beta$ (glycogen synthase kinase $3 \beta$ ). Enfin, miR-199a-3p limite les mécanismes de réparation épithéliale par l'intermédiaire du blocage de l'expression des facteurs de croissance FGF7 (fibroblast growth factor 7) et HGF (hepatocyte growth factor). Autres abréviations: AECl, alveolar epithelial cell of type $1 ; \alpha$ SMA, $\alpha$ smooth muscle actin; $\varepsilon C M(M E C)$, matrice extracellulaire (adapté de [10]). 


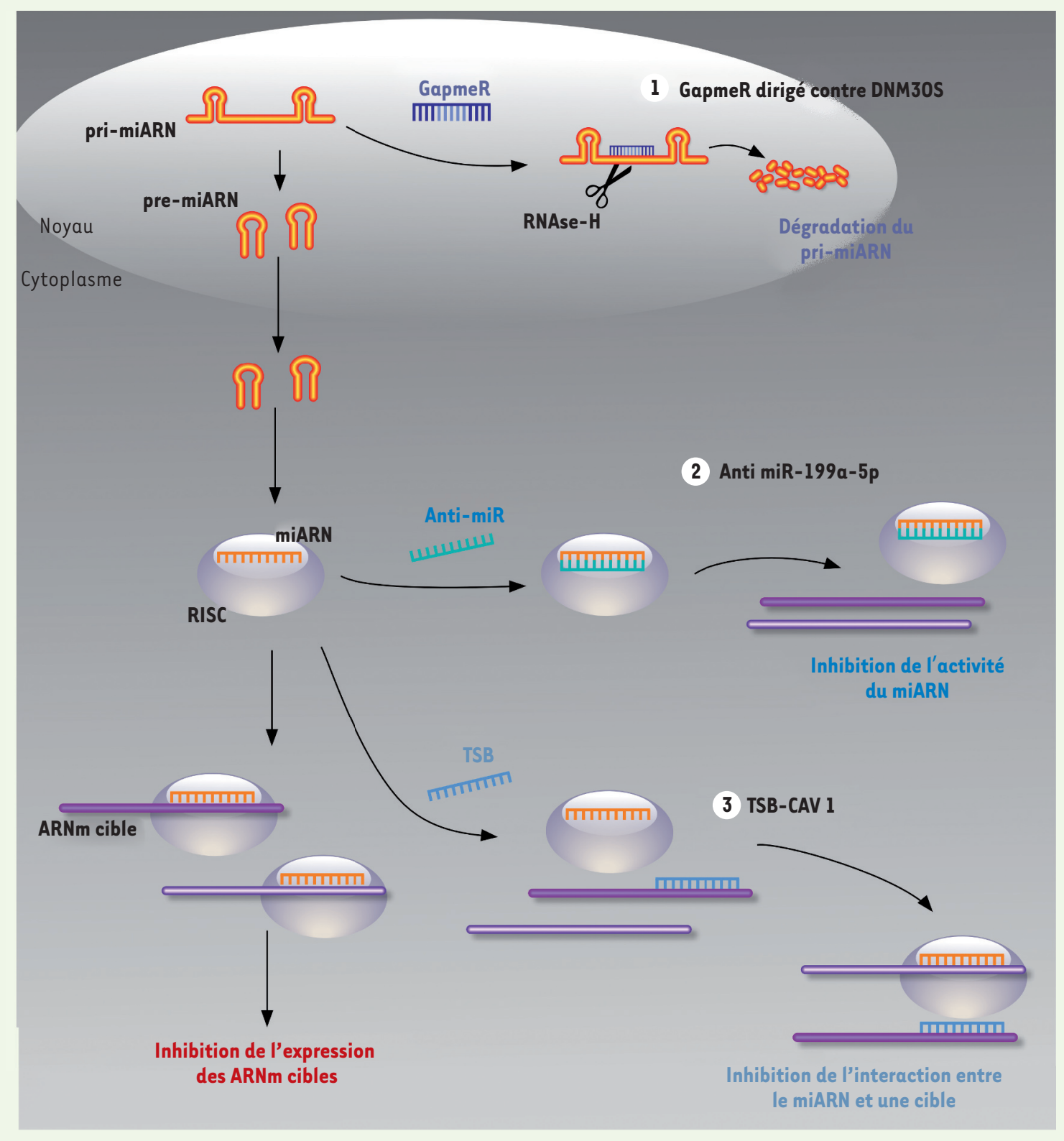

Figure 2. Stratégies permettant d'inhiber l'activité pro-fibrotique de l'ARNnc DNM3OS. Les GapmeR permettent de cibler directement le transcrit primaire au niveau nucléaire et d'induire sa dégradation par l'intermédiaire du recrutement de la RNase $\mathrm{H}$, bloquant ainsi la production des miARN matures (1. Gapmer dirigés contre DNM30S). Les anti-miR sont des oligonucléotides anti-sens complémentaires des miARN matures et forment un duplex les empêchant d'interagir avec leurs ARNm cibles (2. Anti-miR-199a-5p). Les TSB (target site blocker), en se fixant sur un ARNm cible en lieu et place du miARN, empêchent spécifiquement l'interaction du miARN mature avec sa cible (3. TSB-CAV1).

$199 a-3 p$ et miR-214-3p, également surexprimés in vitro en réponse au TGF$\beta$. Ces trois miARN jouent en effet un rôle synergique essentiel dans le processus de fibrogenèse. Ainsi, miR214-3p intervient dans la régulation de la voie de signalisation Wnt / $\beta$-caténine, une voie non-canonique de la réponse des fibroblastes au TGF- $\beta$, tandis que miR-199a-3p est directement impliqué dans l'inhibition des mécanismes de réparation de l'épithélium pulmonaire en inhibant l'expression de deux facteurs de crois- sance essentiels à la cicatrisation, HGF (hepatocyte growth factor) et FGF7 (fibroblast growth factor 7). Au total, ces trois miARN agissent collectivement pour amplifier des mécanismes moléculaires centraux du processus de fibrogenèse (Figure 1) [10]. 
Vers une thérapie ciblant DNM30S ou son contenu miARN?

Etant donné la spécificité d'expression de DNM30S dans les myofibroblastes, son ciblage paraît particulièrement intéressant pour inhiber spécifiquement la voie du TGF- $\beta$ dans ces cellules. Plusieurs stratégies thérapeutiques visant à interférer avec DNM30S ont été développées (Figure 2).

D'une part, nous avons ciblé directement le transcrit non codant DNM30S à l'aide d'oligonucléotides anti-sens de type «GapmeR » qui, grâce à leur structure d'hybride ARN / ADN, induisent le recrutement de la RNAse $\mathrm{H}$ et le clivage nucléaire du transcrit ciblé. Cette stratégie a permis d'inhiber efficacement la surexpression du IncARN et des trois miARN associés dans un modèle murin de fibrose pulmonaire induite par la bléomycine, limitant ainsi le développement des lésions de fibrose. D'autre part, le ciblage de miR-199a-5p, le miARN le plus important dans le contrôle de la voie de signalisation du TGF- $\beta$, à l'aide d'un oligonucléotide complémentaire, a également démontré un effet anti-fibrotique. Enfin, une dernière approche visant à bloquer spécifiquement l'interaction entre miR-199a-5p et l'une de ses cibles, l'ARNm de CAVI, a été testée en utilisant des oligonucléotides de type «TSB » (target site blocker). Cette dernière stratégie a permis de prévenir le développement des lésions de fibrose pulmonaire, ce qui montre que les effets pro-fibrotiques de miR-199a-5p sont essentiellement dus à son interaction avec le transcrit de CAVI.

Nos travaux apportent ainsi un nouvel éclairage sur le rôle des ARNnc dans le processus de fibrose pulmonaire et montrent l'intérêt de leur ciblage thérapeutique. A terme, ces résultats pourraient conduire à l'amélioration de la prise en charge des patients atteints de FPI. De plus, puisque les mécanismes moléculaires décrits dans cette étude concernent également d'autres maladies fibrotiques, il est raisonnable de penser que cette stratégie pourrait aussi s'appliquer à d'autres fibroses d'organes, comme celles touchant le foie, le rein, ou le cœur. $\diamond$

The function of a long non coding RNA decoded in idiopathic pulmonary fibrosis

\section{REMERCIEMENTS}

L'étude a été réalisée grâce aux financements suivants: l'Agence Nationale de la Recherche (ANR) et le «Deutsche Forschungsgemeinschaft» (DFG, Allemagne), la Fondation pour la Recherche Médicale (FRM, prix Mariane Josso attribué à GS), les investissements pour le futur (LABEX SIGNALIFE et FRANCE GENOMIQUE), le Pôle de Recherche Interdisciplinaire sur le Médicament (PRIM), la Société d'Accélération du Transfert de Technologie Nord (SATT Nord), la Fondation Unice (projet AIR), les Fonds de Recherche en Santé Respiratoire et la Fondation du Souffle.

\section{LIENS D'INTÉRÊT}

Les auteurs déclarent n'avoir aucun lien d'intérêt concernant les données publiées dans cet article.

\section{RÉFÉRENCES}

1. Morris K V., Mattick JS. The rise of regulatory RNA. Nat Rev Genet 2014 ; $15: 423-7$

2. Hartmann C, Corre-Menguy F, Boualem A, et al. Les microARN. Med Sci (Paris) 2004 ; $20: 894-98$.

3. Pedrazzini T. Le cœur des ARN non codants. Med Sci (Paris) $2015 ; 31: 261-7$.

4. Pottier N, Cauffiez C, Perrais M, et al. FibromiRs: translating molecular discoveries into new antifibrotic drugs. Trends Pharmacol Sci 2014 ; 35 : 119-26.

5. Chakraborty C, Sharma AR, Sharma G, et al. Therapeutic miRNA and siRNA: moving from bench to clinic as next generation medicine. Mol Ther Nucleic Acids $2017 ; 8$ : 132-43.

6. Lederer DJ, Martinez FJ. Idiopathic pulmonary fibrosis. N Engl J Med 2018 ; 378 : 1811-23.

7. Meng X, Nikolic-Paterson DJ, Lan HY. TGF- $\beta$ : the master regulator of fibrosis. Nat Rev Nephrol 2016; $12: 325-38$.

8. Henaoui IS, Cauffiez C, Aubert $S$, et al. Implication de miR-199a-5p dans la fibrose pulmonaire idiopathique. Med Sci (Paris) 2013 ; $29: 461-3$

9. Lino Cardenas CL, Henaoui IS, Courcot $\varepsilon$, et al. miR$199 a-5 p$ is upregulated during fibrogenic response to tissue injury and mediates TGF $\beta$-induced lung fibroblast activation by targeting caveolin-1. PLoS Genet 2013 ; 9 : el003291.

10. Savary G, Dewaeles $\varepsilon$, Diazzi S, et al. The long noncoding RNA DNM30S is a reservoir of FibromiRs with major functions in lung fibroblast response to TGF- $\beta$ and pulmonary fibrosis. Am J Respir Crit Care Med 2019 ; rccm.201807-12370C.

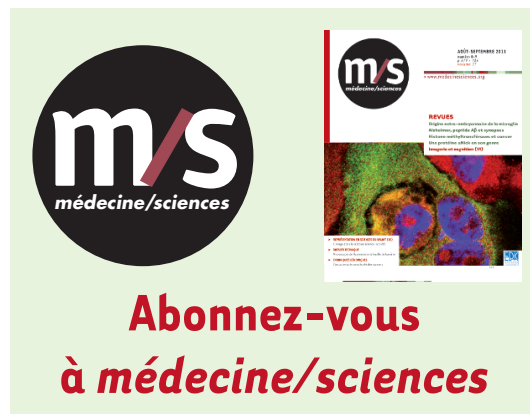

Bulletin d'abonnement page 810 dans ce numéro de $\mathrm{m} / \mathrm{s}$

\section{AMPS (Association Médecine Pharmacie Sciences)}

La période de candidature pour la passerelle permettant d'intégrer directement les études de Santé (Médecine, Pharmacie, Odontologie et Maïeutique) après un diplôme de niveau Bac +5 ou plus approche à grand pas. Pour cette occasion, l'AMPS (Association Médecine Pharmacie Sciences) a le plaisir de vous présenter son guide de candidature au concours passerelle, ainsi que son guide pour préparer sa rentrée directement en $2^{\mathrm{e}}$ ou $3^{\mathrm{e}}$ année des études de Santé, rédigé par des étudiants qui s'y trouvent déjà

Si vous êtes un candidat potentiel, ou que vous en connaissez, n’hésitez pas à les utiliser, ils contiennent théoriquement toutes les informations utiles pour passer du la candidature à la rentrée dans l'année supérieure. Et n'hésitez pas non plus à nous faire part de toutes vos remarques concernant ces guides, nous nous ferons un plaisir de les améliorer.

Enfin, si vous-même avez bénéficié de cette passerelle par le passé, n’hésitez pas à nous contacter pour nous faire part de vos témoignages, vos successeurs vous en remercieront grandement.

Vous trouverez toutes les informations intéressantes sur le site de l'AMPS: https://amps-asso.org/ > Opportunités > Guides Yanis Bendjelal, rédacteur en chef des guides Sciences-Santé de I'AMPS 